\title{
First-principles prediction of one-dimensional giant Rashba splittings in Bi-adsorbed In atomic chains
}

\author{
Tomonori Tanaka* and Yoshihiro Gohda ${ }^{\dagger}$ \\ Department of Materials Science and Engineering, Tokyo Institute of Technology, Yokohama 226-8502, Japan
}

(Received 17 August 2018; revised manuscript received 12 November 2018; published 18 December 2018)

\begin{abstract}
We study Bi-adsorbed In atomic chains on Si(111) in order to design a one-dimensional (1D) Rashba system using first-principles calculations. From the band dispersions and spin textures, we find that this system shows 1D giant Rashba splittings. The Rashba parameters of several structures in this system are comparable with other Rashba systems. Depending on the adsorption structure, this system also shows remarkable features such as a large out-of-plane spin polarization, the reversal of spin polarization in the Rashba bands, and a metal-insulator transition. We propose a mechanism to generate a nondissipative spin current by the gap opening due to an avoided crossing of Rashba bands. This mechanism is suitable for spintronic applications without requiring an external magnetic field.
\end{abstract}

DOI: 10.1103/PhysRevB.98.241409

The Rashba-Bychkov effect (hereafter referred to as simply the Rashba effect) [1] is a momentum-dependent spin splitting of surface or interface bands due to spin-orbit coupling and inversion asymmetry. There has been a growing interest in the effect because it is a candidate for operating principles in spintronic devices [2-5]. Two-dimensional (2D) Rashba systems have been studied extensively [6-15] ever since the initial observations in metal surfaces [16-18]. Furthermore, recently, peculiar spin structures have been found in several Rashba systems [19-23]. The Rashba effect, therefore, has received more attention in terms of fundamental physics as well.

There has been a development in another course regarding this effect-it is the one-dimensional (1D) Rashba effect. The 1D Rashba effect has been observed in atomic chains (nanowires) [24-27] and the surface edge states of Bi [28]. In recent years, it has been reported that many Rashba systems, regardless of whether they are 2D [19,29-32] or 1D systems [24,28], show a finite out-of-plane spin polarization. The peculiar spin polarization cannot be understood by the simple Rashba picture based on a 2D electron gas model [1]. A previous study revealed that the cause of the out-of-plane spin polarization is the in-plane potential gradients due to the anisotropy [33]. The 1D Rashba systems are preferred from the large-spin-splitting point of view; the in-plane potential gradients in the 1D Rashba systems can potentially be larger than those of the 2D systems because of lower symmetry. Moreover, previous studies suggested that the 1D Rashba effect has the possibility of overcoming the limitations of the 2D Rashba effect. A pure spin current using an external

\footnotetext{
*tanaka.t.bj@m.titech.ac.jp

${ }^{\dagger}$ gohda.y.ab@m.titech.ac.jp
}

Published by the American Physical Society under the terms of the Creative Commons Attribution 4.0 International license. Further distribution of this work must maintain attribution to the author(s) and the published article's title, journal citation, and DOI. magnetic field is an example of the nature peculiar to the 1D Rashba effect [34]. The external magnetic field which is applied to a 1D Rashba system removes the degeneracy of the Rashba band at the time-reversal symmetry point. If the Fermi level lies within the gap by applying the voltage, a pure spin current is expected to exist. In addition, this is a nondissipative spin current because of the Fermi surface which has a unidimensional dispersion peculiar to $1 \mathrm{D}$ systems. A nondissipative spin current is an ideal feature for spintronic devices. For these reasons, the 1D Rashba effect becomes increasingly important.

In this Rapid Communication, we predict a candidate for 1D Rashba systems computationally. We study Bi-adsorbed $\mathrm{In} / \mathrm{Si}(111)-4 \times 1$ quasi-1D surfaces using first-principles calculations. Our results reveal that this system exhibits $1 \mathrm{D}$ giant Rashba splittings. The Rashba parameters of this system are comparable with other previously reported Rashba systems. This system also shows a large out-of-plane spin polarization, the reversal of spin polarization in the Rashba bands, and a metal-insulator transition depending on the adsorption structure. We propose a mechanism to generate a nondissipative spin current by the gap opening due to an avoided crossing of Rashba bands. Our mechanism is suitable for spintronic applications, having an advantage that it does not require an external magnetic field to generate a nondissipative spin current.

Our first-principles calculations were based on density functional theory as implemented in the openMX code [35]. We used pseudoatomic orbital basis sets and norm-conserving pseudopotentials. For taking relativistic effects into account, the pseudopotentials were generated based on the Dirac equation, and the spin-orbit coupling for valence electrons is incorporated in self-consistent calculations. The generalized gradient approximation proposed by Perdew, Burke, and Ernzerhof [36] was used to treat the exchange-correlation electron interactions.

The In/Si(111) substrate was simulated as a slab model consisting of three Si bilayers and an In-Si top layer with 
(a)
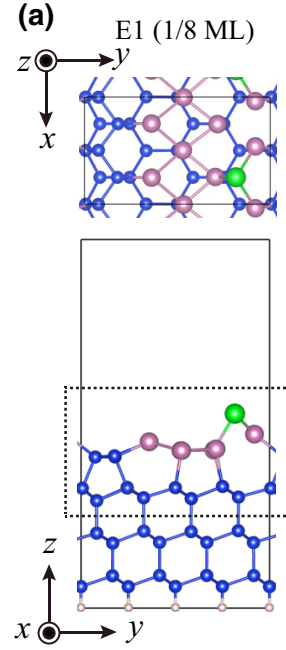

Q1 (1/4 ML)

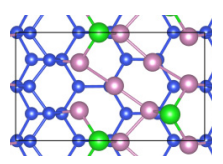

Q2 (1/4 ML)
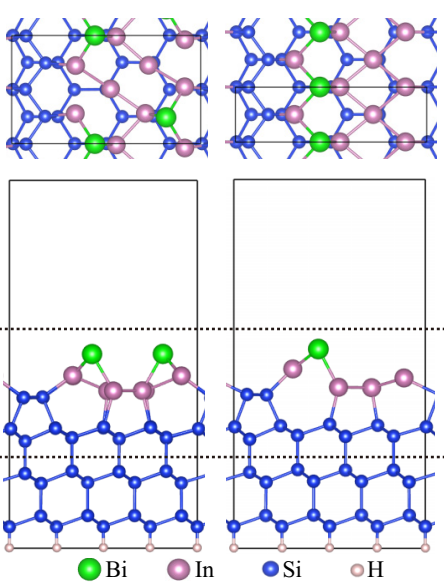

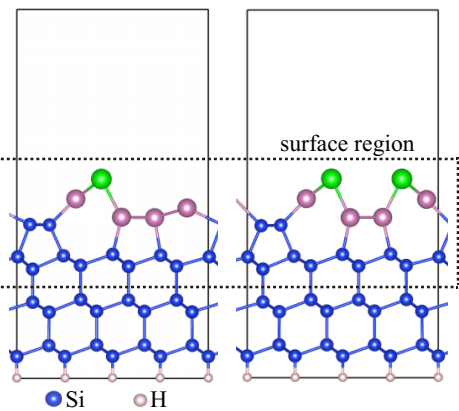

(b)

H1 (1/2 ML)

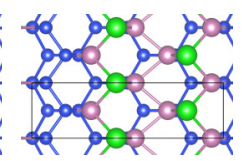

FIG. 1. (a) Stable Bi-adsorbed structures at each Bi coverage. As an exception, the Q2 structure is metastable in the $1 / 4$ ML. Notations "E," "Q," and " $\mathrm{H}$ " in the structure names are assigned based on the Bi coverage, 1/8 (eighth), 1/4 (quarter), and 1/2 (half) ML, respectively. (b) Adsorption free energies per $4 \times 1$ surface as a function of the Bi chemical potential $\Delta \mu_{\mathrm{Bi}}$. a $12-\AA$ vacuum layer in between the slabs. The in-plane lattice constants are $\sqrt{6} a_{0}$ and $a_{0} / \sqrt{2}$ at the $4 \times 1$ unit cell which were determined by optimizing the lattice parameter of the diamond $\mathrm{Si}$ structure $\left(a_{0}=5.48 \AA\right)$. The bottom $\mathrm{Si}$ layer was terminated by hydrogen atoms. As basis sets, $s 2$ configurations were adopted for $\mathrm{H}$ atoms, $s 2 p 2 d 1$ for Si and In atoms, and $s 2 p 2 d 2$ for $\mathrm{Bi}$ atoms. The semicore orbitals of $4 d$ in In as well as $5 d$ in $\mathrm{Bi}$ are treated as valence states. The cutoff radii are 6.0 bohrs for $\mathrm{H}, 11.0$ bohrs for In, 12.0 bohrs for $\mathrm{Bi}, 7.0$ bohrs for $\mathrm{Si}$ in the bottom two bilayers, and 9.0 bohrs for $\mathrm{Si}$ in the surface region, respectively [see also Fig. 1(a)]. We used an energy cutoff of 400 Ry and $23 \times 7 \times 1$ $k$-point grid per first Brillouin zone of the $4 \times 1$ periodic surface cell. The geometry optimization was carried out with the constraint of the bottom Si bilayer coordinates. We optimized structures without considering the spin-orbit coupling. After the optimization, the spin-orbit coupling was taken into account. Convergence criteria for the maximum force on each atom and the total energy are $10^{-4}$ hartree/bohr and $10^{-7}$ hartree, respectively. We also used the VASP code $[37,38]$ with the projector augmented-wave method $[39,40]$ considering the spin-orbit coupling to calculate spin textures. The consistency of the computational results of the two simulation codes was confirmed by the agreement of band dispersions.

We first identified stable atomic structures in various coverages by geometry optimization. Considered coverages are $1 / 2,1 / 4$, and $1 / 8$ monolayers (ML). As initial positions, $\mathrm{Bi}$ atoms are placed at 13 high-symmetry sites proposed by Guerrero-Sánchez and Takeuchi [41]. The stable structures of each coverage are shown in Fig. 1(a). Even though the Q2 structure is not the most stable structure, we display it for reference. We can see that the adsorbed Bi atoms prefer threefold coordinated sites composed of In atoms. This is because a $\mathrm{Bi}$ atom has five valence electrons belonging to $6 s$ and $6 p$ orbitals. The $\mathrm{Bi}$ atom creates the $s p^{3}$ hybridization at the site for bonding with neighboring three In atoms. As a result, these $\mathrm{Bi}$ atoms have no dangling bond.
To compare the stability of the structures having different coverages, we evaluated the adsorption free energy by the following equation [42],

$$
G_{\mathrm{ads}} \simeq E_{\text {slab }}-\left[E_{\text {clean }}+n_{\mathrm{Bi}} \mu_{\mathrm{Bi}}(T, p)\right],
$$

where $E_{\text {slab }}$ and $E_{\text {clean }}$ are the total energies of Bi-adsorbed surface structure and the clean $\operatorname{In} / \mathrm{Si}(111)$ surface, respectively. The number of $\mathrm{Bi}$ atoms per $4 \times 1$ unit cell and the chemical potential of a $\mathrm{Bi}$ atom are represented by $n_{\mathrm{Bi}}$ and $\mu_{\mathrm{Bi}}(T, p)$, respectively. The temperature $(T)$ and pressure $(p)$ dependence of the $G_{\text {ads }}$ is considered only in the term of the Bi chemical potential. The Bi chemical potential $\mu_{\mathrm{Bi}}(T, p)$ consists of two parts: (i) the total energy of a $\mathrm{Bi}$ atom in the relaxed rhombohedral structure $E_{\mathrm{Bi}}^{\text {bulk }}$ and (ii) the temperatureand pressure-dependent part $\Delta \mu_{\mathrm{Bi}}(T, p)$. Hence the Bi chemical potential $\mu_{\mathrm{Bi}}(T, p)$ can be written as

$$
\mu_{\mathrm{Bi}}(T, p)=E_{\mathrm{Bi}}^{\mathrm{bulk}}+\Delta \mu_{\mathrm{Bi}}(T, p) .
$$

Treating the $\Delta \mu_{\mathrm{Bi}}(T, p)$ as a variable, the adsorption free energies are given in Fig. 1(b). In conditions with a low Bi chemical potential, the clean $\mathrm{In} / \mathrm{Si}(111)$ surface is most stable. With increasing the chemical potential, the Q1 and $\mathrm{H} 1$ structures have the most stable range of the Bi chemical potential. From these results, we mainly discuss the electronic structure of the H1 structure as a stable structure. The Q2 structure is also discussed as a reference for the H1 structure, because the behavior of the spin polarization makes a remarkable contrast to the $\mathrm{H} 1$ structure. The $\mathrm{Q} 1$ structure (one of the most stable structures) shows an interesting nature, a metal-insulator transition. However, the results of the Q1 structure are shown in Sec. III of the Supplemental Material [43], because they are different from the main subject of this Rapid Communication.

Band dispersions with spin textures of the Q2 structure are shown in Fig. 2. We found Rashba splitting bands [dashed lines in Fig. 2(a)] crossing the Fermi level. The Rashba bands consist mainly of the $p_{x}$ orbital of the $\mathrm{Bi}$ atom. We confirmed negligibly small dispersion toward a perpendicular 


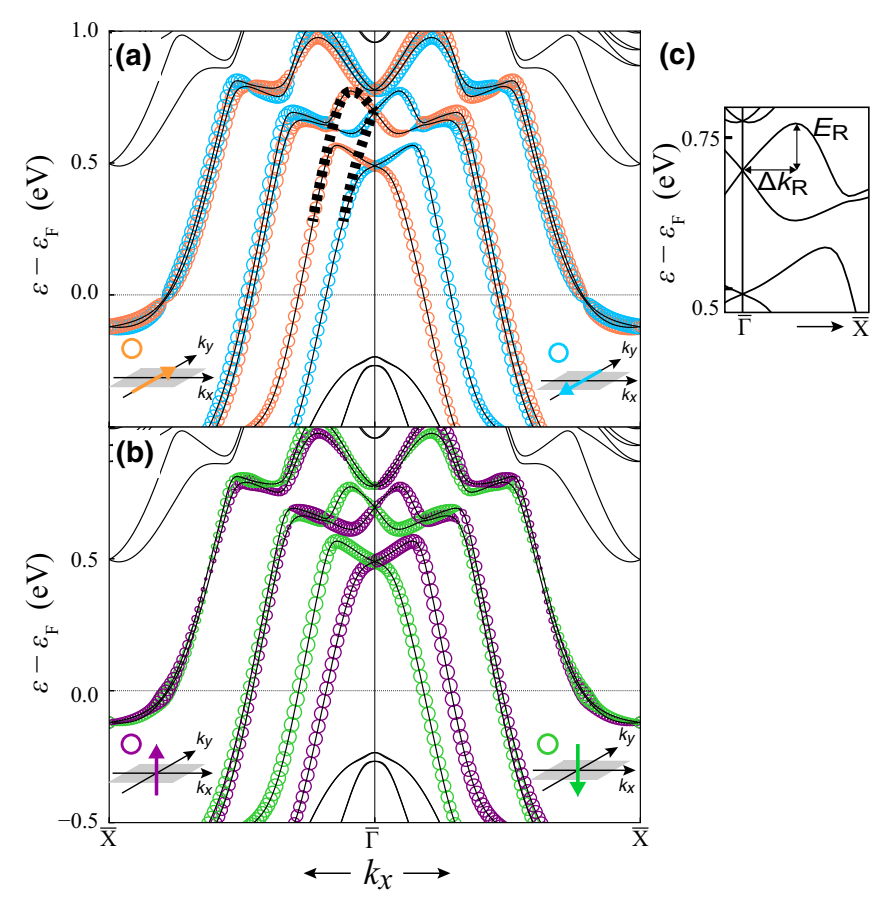

FIG. 2. Spin-polarization components parallel to the (a) $y$ axis and (b) $z$ axis as open circles of the Q2 structure with the band dispersion. The radius of each circle is proportional to the magnitude of the spin component. The circles are drawn only for bands which are crossing $\varepsilon_{\mathrm{F}}$. Spin-polarization components parallel to the $x$ axis are exactly zero on the band path because of the mirror symmetry with respect to the $x$ direction. (c) The definition of the band parameters $E_{\mathrm{R}}$ and $\Delta k_{\mathrm{R}}$ for the Rashba parameter $\alpha_{\mathrm{R}}$.

direction to the atomic chains (i.e., $k_{y}$ direction) on the Fermi surface. The Fermi surface and band characters of the Q2 structure are shown in Sec. I of the Supplemental Material [43]. These results indicate the band splitting is a 1D Rashba splitting. In addition, the Q2 structure shows an out-of-plane spin polarization which is even larger than the in-plane spin polarization [Fig. 2(b)].

As described earlier, the out-of-plane spin polarization was observed in other 1D Rashba systems [24,28]. For example, Okuda et al. determined the cause of the out-of-plane spin polarization in a $\mathrm{Si}(557)$-Au 1D Rashba system as the asymmetry of the partial charge density to the $y$ (in-plane) direction (Fig. 4 in Ref. [24]). These out-of-plane spin polarizations are reasonable, because 1D systems usually show lower in-plane symmetry. As a result, the in-plane potential gradient remains. The Q2 structure has only the mirror symmetry with respect to the $x$ direction. Therefore, the potential gradient to the $y$ direction remains.

We evaluated the Rashba parameter $\alpha_{R}$ of the 1D Rashba splitting in the Q2 structure. The Rashba parameter is used as an indicator of the magnitude of a Rashba splitting. The Rashba parameter is defined as $\alpha_{\mathrm{R}}=2 E_{\mathrm{R}} / \Delta k_{\mathrm{R}}$, where $E_{\mathrm{R}}$ is the Rashba energy and $\Delta k_{\mathrm{R}}$ is the Rashba momentum offset [12]. These two band parameters are illustrated in Fig. 2(c). Following the definition, the $\alpha_{\mathrm{R}}$ of the Q2 structure is $2.1 \mathrm{eV} \AA$.

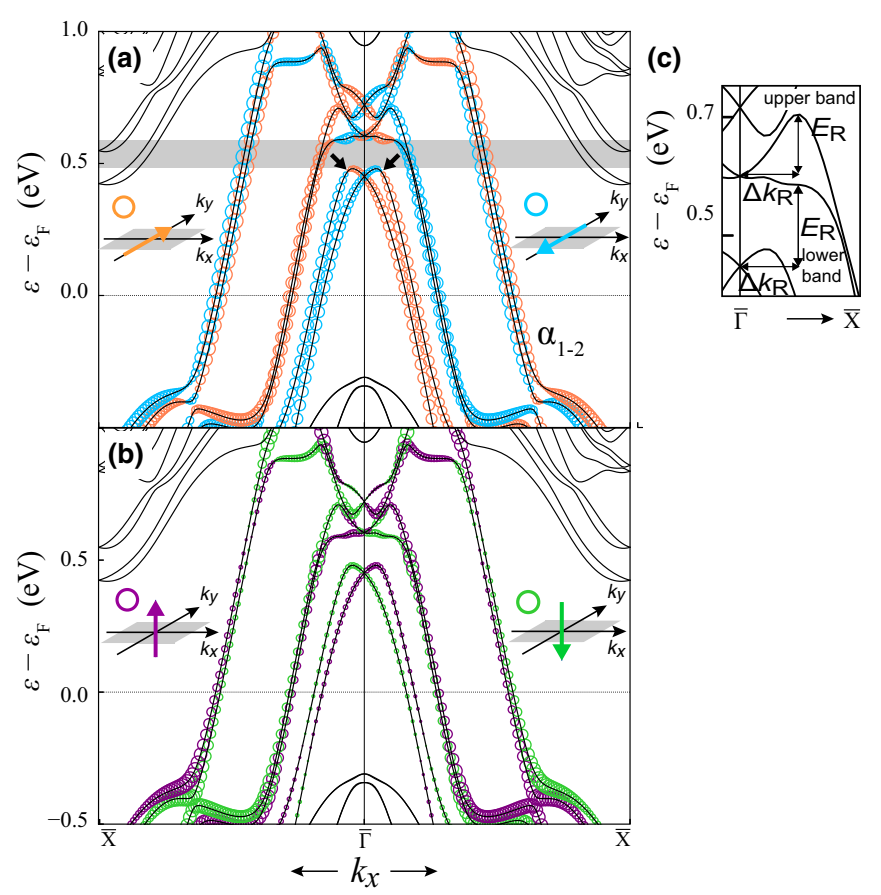

FIG. 3. Spin-polarization components parallel to the (a) $y$ axis and (b) $z$ axis as open circles of the $\mathrm{H} 1$ structure with the band dispersion. The radius of each circle is proportional to the magnitude of the spin component. (c) The definition of the band parameters $E_{\mathrm{R}}$ and $\Delta k_{\mathrm{R}}$ for the Rashba parameter $\alpha_{\mathrm{R}}$.

Band dispersions with spin textures of the $\mathrm{H} 1$ structure are shown in Fig. 3. Unlike a conventional Rashba splitting, the direction of the spin polarization reverses near the $\bar{\Gamma}$ point [indicated by black arrows in Fig. 3(a)]. To clarify the origin of the spin reversal, we constructed a hypothetical surface model shown in Fig. 4(a). We call it an H1-like undistorted structure. In the H1 structure, the surface atoms (In and $\mathrm{Bi}$ ) are slightly distorted due to the effect of Si stacking inside the substrate. In other words, the influence of the potential from the $\mathrm{Si}$ atoms inside the substrate is different on both sides of the In atomic chain. As a result, the atoms on both sides of the In atomic chain are inequivalent. The H1-like undistorted structure was constructed to investigate the effect of the distortion by comparing with the H1 structure. The H1-like structure was obtained according to the following procedures. First, the Si underlayers were removed from the $\mathrm{H} 1$ structure. The new bottom $\mathrm{Si}$ atoms were terminated by hydrogen atoms. Next, the coordinates of the atoms on both sides of the In atomic chain were adjusted to be equivalent. Finally, geometry optimization was carried out while maintaining the equivalence.

Spin textures of the H1-like structure are shown in Figs. 4(b) and 4(c). We can see that the spin polarizations are reversed in the vicinity of the region where the avoided crossing of the Rashba bands occurs [red arrows in Fig. 4(b)]. To clarify the cause of the spin-reversal phenomenon, we classified four Rashba bands into two irreducible representations with a group-theoretical analysis. Details of the analysis are shown in Sec. II of the Supplemental Material [43]. The analysis revealed that the avoided-crossing Rashba bands, which 
(a)
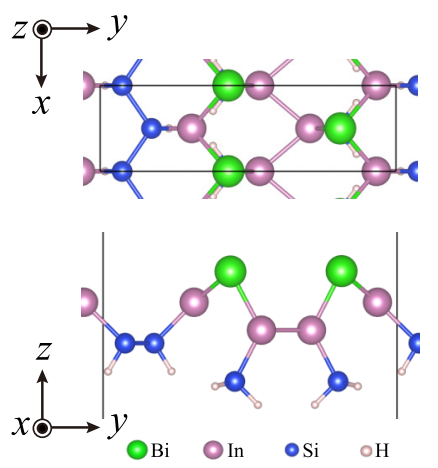

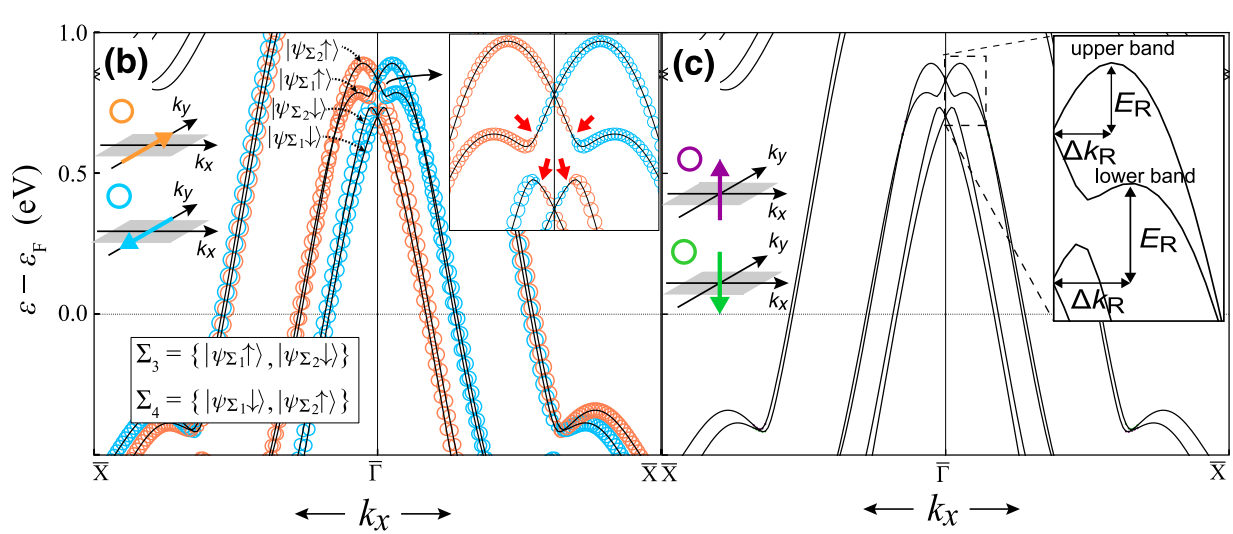

FIG. 4. (a) Atomic structure of the H1-like undistorted structure. Spin-polarization components parallel to the (b) $y$ axis and (c) $z$ axis as open circles of the structure with the band dispersion. The radius of each circle is proportional to the magnitude of the spin component. The labels for the four Rashba bands and the classification into the irreducible representations $\left(\Sigma_{3}\right.$ and $\left.\Sigma_{4}\right)$ are also shown in (b). The inset in (c) shows the definition of the band parameters $E_{\mathrm{R}}$ and $\Delta k_{\mathrm{R}}$ for the Rashba parameters $\alpha_{\mathrm{R}}$.

are labeled $\left|\psi_{\Sigma_{1}} \uparrow\right\rangle$ and $\left|\psi_{\Sigma_{2}} \downarrow\right\rangle$ in Fig. 4(b), belong to the same irreducible representation $\Sigma_{3}$. It means that the energy gap around the spin-reversal point is due to the hybridization of the Rashba bands. In the case of the H1 structure, we consider that the degree of hybridization is changed by the structure distortion. As a result, the width of the gap becomes wider than that of the H1-like structure.

The spin-reversal phenomenon in Rashba bands is worthy of special remark in terms of the generation of the spin current. If the Fermi level lies within the gap around the spin-reversal point by applying the voltage, the spin current can exist. The energy range of the gap is shown as a horizontal shaded gray background in Fig. 3(a). Furthermore, if we ignore the outer two bands in the H1 structure $\left[\alpha_{1-2}\right.$ in Fig. 3(a)], the spin current has a nondissipative nature. The schematic concept is shown in Fig. 5; the hybridization of the crossing Rashba bands yields the energy gap which generates the nondissipative spin current. This condition prohibits backscattering, because the Rashba states with opposite sign
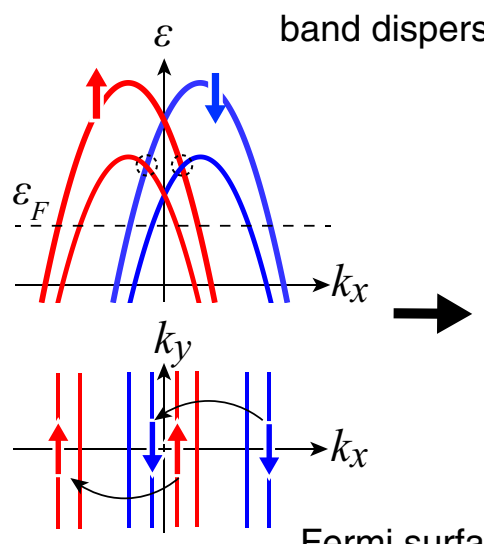
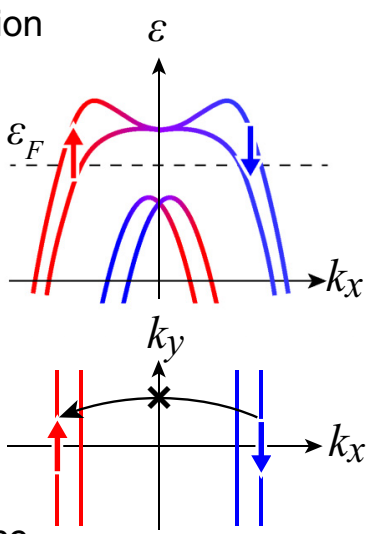

FIG. 5. Mechanism to generate a nondissipative spin current without requiring an external magnetic field. The crossing points (dashed circles) are repelled due to hybridization between the Rashba bands. If the Fermi level lies within the gap by applying the voltage, the nondissipative spin current is expected to exist. momentum are spin polarized oppositely (the right side of Fig. 5). We would like to emphasize the significant difference between this mechanism and a previously proposed one [34]. The previous system requires an external magnetic field to open the energy gap which generates a nondissipative spin current. In contrast, our mechanism does not require an external magnetic field to open the gap. This is a great advantage for energy efficiency and the simplification of spintronic devices.

We have to state a problem in our mechanism for the nondissipative nature of the spin current. When the structure distortion which is the cause of widening the gap involves a change in symmetry, an out-of-plane spin polarization could exist. This out-of-plane polarization is also seen in the H1 structure [Fig. 3(b)]. The induced out-of-plane spin polarization deviates from the complete antiparallel spin polarization of electrons with opposite sign momentum. The deviation allows a slight backscattering of electrons $[44,45]$. However, our group-theoretical analysis presents a way to avoid this problem. As mentioned above, the gap around the spinreversal point is due to hybridization of the Rashba states. If one adjusts the degree of hybridization with appropriate symmetry, the gap becomes wider without an out-of-plane spin polarization (an artificial structure for testing this suggestion is shown in Fig. S6). Therefore, it is attractive to explore systems that satisfy the above conditions in other 1D systems.

A similar spin-reversal phenomenon was reported in a $\mathrm{Bi} / \mathrm{Cu}(111)$ surface alloy [20], where the spin-reversal mechanism is the change of the character of orbitals within the Rashba band. In contrast, the present $\mathrm{Bi}$ on $\mathrm{In} / \mathrm{Si}(111)$ system exhibits spin reversal due to the hybridization of two Rashba bands consisting of $p_{x}$ orbitals of two $\mathrm{Bi}$ atoms in the unit cell, as is demonstrated in Sec. II of the Supplemental Material [43].

We evaluated the Rashba parameters of the H1 and H1like structure as is the case in the Q2 structure. Obtaining $E_{\mathrm{R}}$ and $\Delta k_{\mathrm{R}}$ is illustrated in Figs. 3(c) and 4(c) in order to determine $\alpha_{\mathrm{R}}$. The Rashba parameters were evaluated to be 2.7 and $3.5 \mathrm{eV} \AA$ for upper and lower Rashba bands in the $\mathrm{H} 1$ structure, respectively. We also evaluated the Rashba parameters of the H1-like structure: 2.8 and $3.1 \mathrm{eV} \AA$ for the 
TABLE I. Parameters characterizing spin splitting: Rashba energy $\left(E_{\mathrm{R}}\right)$, Rashba momentum offset $\left(\Delta k_{\mathrm{R}}\right)$, and Rashba parameter $\left(\alpha_{\mathrm{R}}\right)$ for the present study, together with previously reported Rashba systems.

\begin{tabular}{lrlc}
\hline \hline Rashba systems & $E_{\mathrm{R}}(\mathrm{meV})$ & $\Delta k_{\mathrm{R}}\left(\AA^{-1}\right)$ & $\alpha_{\mathrm{R}}(\mathrm{eV} \AA)$ \\
\hline Bi on In/Si(111)-4 × 1 (1D) & & & \\
Q2 & 78.2 & 0.073 & 2.1 \\
H1 upper band & 106 & 0.079 & 2.7 \\
H1 lower band & 139 & 0.079 & 3.5 \\
H1-like upper band & 83.9 & 0.054 & 2.8 \\
H1-like lower band & 59.6 & 0.042 & 3.1 \\
Bi/InAs(110) (1D) [27] & 290 & 0.105 & 5.5 \\
Pt/Si(111) (1D) [25] & 81 & 0.12 & 1.36 \\
Bi(111) edge (1D) [28] & 68 & 0.17 & 0.80 \\
Bi/Ag(111) (2D) [8] & 200 & 0.13 & 3.05 \\
BiTeI bulk (3D) [14] & 100 & 0.052 & 3.8 \\
\hline \hline
\end{tabular}

upper and lower Rashba bands, respectively. These values are comparable with other Rashba systems (Table I).

Compared with the Q2 structure, the out-of-plane spin polarization of the $\mathrm{H} 1$ structure is small. We consider that the cause of the small out-of-plane spin polarization is the offset of the potential gradient parallel to the $y$ direction due to the placement of the $\mathrm{Bi}$ atoms; the $\mathrm{Bi}$ atoms of the $\mathrm{H} 1$ structure are placed on both sides of the atomic chains. It is also understood from the result of the spin polarization of the H1-like structure [Fig. 4(c)]. There is no out-of-plane spin polarization in the $\mathrm{H} 1$-like structure, because the in-plane potential gradient is completely canceled out as the atoms on both sides of the atomic chain were made equivalent. The result of the H1-like structure supports the hypothesis that the $\mathrm{Bi}$ atoms on both sides offset the in-plane potential gradient.

In conclusion, we have predicted 1D giant Rashba splittings in Bi-adsorbed In atomic chains. The Rashba parameters are comparable with other 1D Rashba systems examined previously. This system also shows a large out-of-plane spin polarization (Q2 structure), spin reversal in the Rashba bands (H1 structure), and a metal-insulator transition (Q1 structure). In particular, the spin-reversal phenomenon by the gap opening due to the avoided crossing of Rashba bands suggests a mechanism to generate a nondissipative spin current without requiring the external magnetic field. Our study should be important for energy-efficient spintronic applications and stimulating further exploration of different 1D Rashba systems.

This work was supported in part by MEXT as a social and scientific priority issue CDMSI to be tackled by using the post-K computer and the Elements Strategy Initiative Project as well as KAKENHI Grant No. 17K04978. We are grateful to Y. Tatetsu and A. Terasawa for proofreading of the manuscript. The calculations were partly carried out by using supercomputers at ISSP, The University of Tokyo, and TSUBAME, Tokyo Institute of Technology as well as the K computer, RIKEN (Projects No. hp170269 and No. hp180206).
[1] Y. A. Bychkov and E. I. Rashba, J. Phys. C: Solid State Phys. 17, 6039 (1984).

[2] S. Datta and B. Das, Appl. Phys. Lett. 56, 665 (1990).

[3] J. Nitta, T. Akazaki, H. Takayanagi, and T. Enoki, Phys. Rev. Lett. 78, 1335 (1997).

[4] T. Koga, J. Nitta, H. Takayanagi, and S. Datta, Phys. Rev. Lett. 88, 126601 (2002).

[5] D. D. Awschalom and M. E. Flatté, Nat. Phys. 3, 153 (2007).

[6] D. Pacilé, C. R. Ast, M. Papagno, C. Da Silva, L. Moreschini, M. Falub, A. P. Seitsonen, and M. Grioni, Phys. Rev. B 73, 245429 (2006).

[7] T. Nakagawa, O. Ohgami, Y. Saito, H. Okuyama, M. Nishijima, and T. Aruga, Phys. Rev. B 75, 155409 (2007).

[8] C. R. Ast, J. Henk, A. Ernst, L. Moreschini, M. C. Falub, D. Pacilé, P. Bruno, K. Kern, and M. Grioni, Phys. Rev. Lett. 98, 186807 (2007).

[9] T. Kadono, K. Miyamoto, R. Nishimura, K. Kanomaru, S. Qiao, K. Shimada, H. Namatame, A. Kimura, and M. Taniguchi, Appl. Phys. Lett. 93, 252107 (2008).

[10] S. Hatta, T. Aruga, C. Kato, S. Takahashi, H. Okuyama, A. Harasawa, T. Okuda, and T. Kinoshita, Phys. Rev. B 77, 245436 (2008)

[11] I. Gierz, T. Suzuki, E. Frantzeskakis, S. Pons, S. Ostanin, A. Ernst, J. Henk, M. Grioni, K. Kern, and C. R. Ast, Phys. Rev. Lett. 103, 046803 (2009).

[12] L. Moreschini, A. Bendounan, H. Bentmann, M. Assig, K. Kern, F. Reinert, J. Henk, C. R. Ast, and M. Grioni, Phys. Rev. B 80, 035438 (2009).
[13] S. Hatta, T. Aruga, Y. Ohtsubo, and H. Okuyama, Phys. Rev. B 80, 113309 (2009).

[14] K. Ishizaka, M. S. Bahramy, H. Murakawa, M. Sakano, T. Shimojima, T. Sonobe, K. Koizumi, S. Shin, H. Miyahara, A. Kimura, K. Miyamoto, T. Okuda, H. Namatame, M. Taniguchi, R. Arita, N. Nagaosa, K. Kobayashi, Y. Murakami, R. Kumai, Y. Kaneko, Y. Onose, and Y. Tokura, Nat. Mater. 10, 521 (2011).

[15] A.V. Matetskiy, S. Ichinokura, L.V. Bondarenko, A.Y. Tupchaya, D.V. Gruznev, A.V. Zotov, A. A. Saranin, R. Hobara, A. Takayama, and S. Hasegawa, Phys. Rev. Lett. 115, 147003 (2015).

[16] S. LaShell, B. A. McDougall, and E. Jensen, Phys. Rev. Lett. 77, 3419 (1996).

[17] M. Hochstrasser, J. G. Tobin, E. Rotenberg, and S. D. Kevan, Phys. Rev. Lett. 89, 216802 (2002).

[18] Y. M. Koroteev, G. Bihlmayer, J. E. Gayone, E. V. Chulkov, S. Blügel, P. M. Echenique, and P. Hofmann, Phys. Rev. Lett. 93, 046403 (2004).

[19] K. Sakamoto, T. Oda, A. Kimura, K. Miyamoto, M. Tsujikawa, A. Imai, N. Ueno, H. Namatame, M. Taniguchi, P. E. J. Eriksson, and R. I. G. Uhrberg, Phys. Rev. Lett. 102, 096805 (2009).

[20] H. Mirhosseini, J. Henk, A. Ernst, S. Ostanin, C.-T. Chiang, P. Yu, A. Winkelmann, and J. Kirschner, Phys. Rev. B 79, 245428 (2009).

[21] K. Sakamoto, H. Kakuta, K. Sugawara, K. Miyamoto, A. Kimura, T. Kuzumaki, N. Ueno, E. Annese, J. Fujii, A. 
Kodama, T. Shishidou, H. Namatame, M. Taniguchi, T. Sato, T. Takahashi, and T. Oguchi, Phys. Rev. Lett. 103, 156801 (2009).

[22] S. D. Stolwijk, K. Sakamoto, A. B. Schmidt, P. Krüger, and M. Donath, Phys. Rev. B 91, 245420 (2015).

[23] E. Annese, T. Kuzumaki, B. Müller, Y. Yamamoto, H. Nakano, H. Kato, A. Araki, M. Ohtaka, T. Aoki, H. Ishikawa, T. Hayashida, J. R. Osiecki, K. Miyamoto, Y. Takeichi, A. Harasawa, K. Yaji, T. Shirasawa, K. I. Nittoh, W. Yang, K. Miki, T. Oda, H. W. Yeom, and K. Sakamoto, Phys. Rev. Lett. 117, 016803 (2016).

[24] T. Okuda, K. Miyamoto, Y. Takeichi, H. Miyahara, M. Ogawa, A. Harasawa, A. Kimura, I. Matsuda, A. Kakizaki, T. Shishidou, and T. Oguchi, Phys. Rev. B 82, 161410 (2010).

[25] J. Park, S. W. Jung, M.-C. Jung, H. Yamane, N. Kosugi, and H. W. Yeom, Phys. Rev. Lett. 110, 036801 (2013).

[26] M. Kopciuszyński, M. Krawiec, R. Zdyb, and M. Jałochowski, Sci. Rep. 7, 46215 (2017).

[27] T. Nakamura, Y. Ohtsubo, Y. Yamashita, S. I. Ideta, K. Tanaka, K. Yaji, A. Harasawa, S. Shin, F. Komori, R. Yukawa, K. Horiba, H. Kumigashira, and S. I. Kimura, Phys. Rev. B 98, 075431 (2018).

[28] A. Takayama, T. Sato, S. Souma, T. Oguchi, and T. Takahashi, Phys. Rev. Lett. 114, 066402 (2015).

[29] M.-H. Liu and C.-R. Chang, Phys. Rev. B 80, 241304 (2009).

[30] A. Takayama, T. Sato, S. Souma, and T. Takahashi, Phys. Rev. Lett. 106, 166401 (2011).

[31] J. Ibañez-Azpiroz, A. Eiguren, and A. Bergara, Phys. Rev. B 84, 125435 (2011).

[32] J. Ibañez-Azpiroz, A. Bergara, E. Y. Sherman, and A. Eiguren, Phys. Rev. B 88, 125404 (2013).

[33] J. Henk, A. Ernst, and P. Bruno, Phys. Rev. B 68, 165416 (2003).

[34] C. H. L. Quay, T. L. Hughes, J. A. Sulpizio, L. N. Pfeiffer, K. W. Baldwin, K. W. West, D. Goldhaber-Gordon, and R. de Picciotto, Nat. Phys. 6, 336 (2010).

[35] T. Ozaki, Phys. Rev. B 67, 155108 (2003).

[36] J. P. Perdew, K. Burke, and M. Ernzerhof, Phys. Rev. Lett. 77, 3865 (1996).

[37] G. Kresse and J. Furthmüller, Comput. Mater. Sci. 6, 15 (1996).
[38] G. Kresse and J. Furthmüller, Phys. Rev. B 54, 11169 (1996).

[39] P. E. Blöchl, Phys. Rev. B 50, 17953 (1994).

[40] G. Kresse and D. Joubert, Phys. Rev. B 59, 1758 (1999).

[41] J. Guerrero-Sánchez and N. Takeuchi, Appl. Surf. Sci. 385, 318 (2016).

[42] K. Reuter and M. Scheffler, Phys. Rev. B 65, 035406 (2001).

[43] See Supplemental Material at http://link.aps.org/supplemental/ 10.1103/PhysRevB.98.241409 for (I) orbital contributions of Rashba bands, (II) group-theoretical analysis for the origin of the gap around the spin-reversal point, and (III) band dispersion and spin texture of the Q1 structure, which includes Refs. [46-54].

[44] P. Roushan, J. Seo, C. V. Parker, Y. S. Hor, D. Hsieh, D. Qian, A. Richardella, M. Z. Hasan, R. J. Cava, and A. Yazdani, Nature (London) 460, 1106 (2009).

[45] T. Zhang, P. Cheng, X. Chen, J.-F. Jia, X. Ma, K. He, L. Wang, H. Zhang, X. Dai, Z. Fang, X. Xie, and Q.-K. Xue, Phys. Rev. Lett. 103, 266803 (2009).

[46] T. Inui, Y. Tanabe, and Y. Onodera, Group Theory and Its Applications in Physics (Springer, New York, 1996).

[47] H. W. Yeom, S. Takeda, E. Rotenberg, I. Matsuda, K. Horikoshi, J. Schaefer, C. M. Lee, S. D. Kevan, T. Ohta, T. Nagao, and S. Hasegawa, Phys. Rev. Lett. 82, 4898 (1999).

[48] J. Guo, G. Lee, and E. W. Plummer, Phys. Rev. Lett. 95, 046102 (2005).

[49] G. Lee, J. Guo, and E. W. Plummer, Phys. Rev. Lett. 95, 116103 (2005)

[50] S. J. Park, H. W. Yeom, J. R. Ahn, and I.-W. Lyo, Phys. Rev. Lett. 95, 126102 (2005).

[51] C. González, J. Ortega, and F. Flores, New J. Phys. 7, 100 (2005).

[52] C. González, F. Flores, and J. Ortega, Phys. Rev. Lett. 96, 136101 (2006).

[53] C. González, J. Guo, J. Ortega, F. Flores, and H. H. Weitering, Phys. Rev. Lett. 102, 115501 (2009).

[54] Y. J. Sun, S. Agario, S. Souma, K. Sugawara, Y. Tago, T. Sato, and T. Takahashi, Phys. Rev. B 77, 125115 (2008). 